\title{
Optimal Coordination of Overcurrent Relays in Microgrids using a Metaheuristic Approach
}

\author{
Sergio D. Saldarriaga-Zuluaga ${ }^{1}$, Carlos D. Zuluaga ${ }^{2}$, Nicolás Muñoz-Galeano ${ }^{3}$, Jesús M. López-Lezama ${ }^{4}$ \\ ${ }^{1}$ Institución Universitaria Pascual Bravo, Facultad de Ingeniería, Calle 73 no 73A-226, Medellín, Colombia, \\ and with the Department of Electrical Engineering, \\ University of Antioquia (UdeA) \\ ${ }^{2}$ Institución Universitaria Pascual Bravo, Facultad de Ingeniería, Calle 73 no 73A-226, Medellín, Colombia, \\ ${ }^{3}$ Department of Electrical Engineering, University of Antioquia (UdeA), Calle 67 No 53-108, Medellín, Colombia \\ ${ }^{4}$ Department of Electrical Engineering, University of Antioquia (UdeA), Calle 67 No 53-108, Medellín, Colombia
}

ORCIDs: 0000-0002-9134-8576 (Sergio), 0000-0002-1196-2227 (Carlos), 0000-0003-1407-5559 (Nicolás), 0000-0002-2369-6173 (Jesús)

\begin{abstract}
One of the key technical issues in the practical implementation of microgrids is their protection coordination scheme. The protection coordination of a microgrid must be able to meet the basic protection requirements of selectivity, sensitivity and reliability for several operation modes or topologies. This paper presents an optimal coordination approach of overcurrent relays in microgrids that can be used for several operation modes or topologies. The protection coordination is solved by a genetic algorithm (GA). Several tests were performed on a benchmark IEC microgrid showing the applicability and effectiveness of the proposed approach. The proposed GA is able to find the protection coordination of overcurrent relays for several operative topologies guaranteeing coordination between main and backup relays.
\end{abstract}

Keywords: Genetic algorithms, microgrids, overcurrent relays, protection coordination.

\section{INTRODUCTION}

Traditional distribution networks exhibit a radial top-down architecture in which all loads are supplied through a main substation which acts as the single source of the network. This topology offers low resilience to faults, since a single failure at the transmission level may cause the loss of many distribution systems. Also, traditional grids require high investment, increase transmission line loses and are responsible for higher emissions of greenhouse gases than modern networks [1]. Within the last two decades, technological advances in small-scale generation technologies, as well as a renewed ecological awareness resulted in the so called active networks. These are distribution networks that allow the presence of distributed generation (DG). The evolution of these networks are what is currently known as microgrids [2]. A microgrid can be defined as a set of distributed energy resources (DERs), storage devices and loads, connected to a main grid through a controllable switch that provides electric power to a local community [3]. The advantages, impacts and challenges offered by microgrids can be analyzed considering different points of view [4]. From the perspective of the network operator, a microgrid can be seen as a controllable unit that can be exploited as a concentrated load. In this case, the excess/lack of generation within the microgrid can be modeled from the power system standpoint as a positive/negative power injection at the node of the substation. From the customers' standpoint, microgrids can be designed to provide electricity with better local voltages, lower energy losses, higher efficiency and in some cases, uninterruptible power supply [5]. From an environmental point of view, DG microgrids contribute to the reduction of environmental pollution and global warming due to the incorporation of renewable sources of energy [6].

When a microgid has sufficient generation to locally supply its load, it can operate connected to the conventional power gird or in islanded mode without incurring in load shedding. Furthermore, if a microgrid has excess generation capacity it can provide the generation surplus to the transmission system [7]. Therefore, the two main operation modes of a microgrid are grid connected and islanded mode. During grid connected mode, the microgrid can receive power from both, the distributed generation (DG) and the main grid; conversely, in islanded mode the microgird only receives power form the DG. In this case, load/generation shedding must be carried out to compensate power imbalance.

Despite of the advantages that microgrids offer, they have not been massively implemented due to a number of technical challenges. Some of them include power quality, voltage and frequency control, energy management, system stability and system protection. One of the main challenges in microgrids operation is their protection coordination. The main objective of a microgrid is to provide reliable energy to its customers. However, traditional protection schemes designed for radial 
power flow are not suitable in microgrids [8]. This is due to a series of factors such as bidirectional power flows, dynamic characteristics of micro sources, limitation of fault currents during islanded mode as well as the number and type of DG technologies within the microgrid [9].

Several studies have been conducted regarding protection coordination in microgirds. In [10], the authors proposed a protection coordination index suitable for looped distribution systems with DG. A nonlinear optimization problem was proposed for determining such index considering variations in the penetration levels of DG. In [11], the protection coordination problem was solved to determine the optimal relay settings considering single contingencies in lines or DG units and taking into account both the grid connected and isolated operation modes. In [12], an online adaptive protection coordination was proposed. In this case, the authors used numerical directional overcurrent relays (OCRs) which were coordinated by an interior point optimization solver. Several metaheuristic techniques have also been proposed to tackle the optimal coordination problem in microgrids. In [13], the authors implemented a metaheuristic approach known as invasive weed optimization (IWO) to solve the coordination of directional OCRs in microgrids with high penetration of DG. The optimal coordination of directional OCR was also proposed in [14] using a hybrid particle swarm optimization (PSO) technique. In [15], the authors implemented a teaching learning-based optimization (TLBO) for setting of overcurrent relays with the aim of reducing the incidence of nuisance tripping in network topology changes. In [16], a multi-objective optimization algorithm was presented for the coordination of OCRs in microgrids based on PSO.

This paper aims to contribute to the discussion of the application of metaheuristic techniques to the optimal coordination of OCRs in microgrids. In this case, the optimal coordination is solved through by a GA. The main advantage of the proposed approach lies on its adaptability to different network topologies guaranteeing coordination between main and backup relays in all operative scenarios. This paper is organized as follows. Section II presents the mathematical formulation of the problem, Section III describes the solution technique, Section IV presents the tests and results on a benchmark IEC microgrid; finally, Section V presents the conclusions of the paper.

\section{MATHEMATICAL FORMULATION}

The mathematical formulation of the coordination of directional OCRs in microgrids is presented in equations (1) (6). The model aims to minimize the total operation time of the relays, ensuring coordination between the main and backup relays.

$$
\min \sum_{i=1}^{m} \sum_{f=1}^{n} t_{i f}
$$

$$
\begin{gathered}
t_{i f}-t_{i j} \geq M C T \\
t_{\text {imin }} \leq t_{i f} \leq t_{\text {imax }} \\
T M S_{\text {imin }} \leq T M S_{i} \leq T M S_{\text {imax }} \\
\text { ipickup }_{\text {imin }} \leq \text { ipickup }_{i} \leq \text { ipickup }_{\text {imax }} \\
t_{i f}=\frac{\text { AxTMS }_{i}}{\left(\frac{I_{f i}}{\text { ipickup }_{i}}\right)^{B}-1}
\end{gathered}
$$

Equation (1) represents the objective function, where $t_{i f}$ is the operation time of relay $i$ for a fault $f ; m$ is the number of relays within the microgrid and $n$ is the number of faults in the system. Equation (2) represents the minimum coordination time between the main relay and the backup relay for each of the faults. In this case, $t_{i f}$ and $t_{j f}$ are the operating time of the main relay $i$ and backup $j$, respectively when the fault $f$ occurs. The constant $M C T$ represents the coordination time considered. Equation (3) represents the minimum and maximum limits of the relay operating time, denoted as $t_{\text {imin }}$ and $t_{\text {imax }}$, respectively. In (4), $T M S_{i}$ is the dial of the operation curve of the relay; this constraint represents its minimum and maximum limits. Equation (5) defines the minimum and maximum limits of the pickup current of relay $i$ (ipickup $_{i}$ ). Equation (6) represents the operation time $t_{i f}$ of relay $i$ when fault $f$ takes place. Finally, the variable $I_{f i}$ represents the fault current seen by relay $i$ when the fault $f$ occurres. Equation (6) represents the characteristic operating curve of each relay, the particularity of the curve is defined by means of constants A and B. The value of such constants depends on the selected standard that can be IEEE or IEC and the type of curve selected. In this case, a normally inverse type curve of the IEC standard was considered. For this curve the constants $\mathrm{A}$ and $\mathrm{B}$ are 0.14 and 0.02 respectively.

\section{METHODOLOGY}

The model given by (1)-(6) was solved using a GA. These algorithms have proven to be effective when dealing with non-linear and non-convex optimization problems such as the one being addressed. GAs are based on the principle of Darwinian evolution, in which the fittest individuals are more likely to pass on their genes to the next generation. A GA starts with an initial population o set of solution candidates which must pass through the stages of fitness evaluation, selection, crossover and mutation. Every cycle of these stages is called a generation. The algorithm stops after a given number of generations or when a certain number of generations have been evaluated without any improvement of the objective function. Figure 1 depicts the flowchart of the proposed GA. 


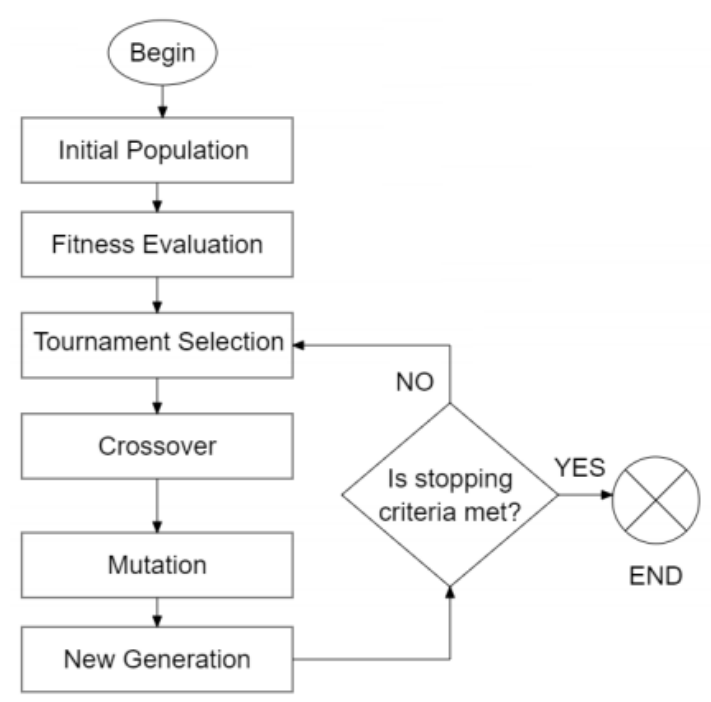

Fig. 1. Flowchart of the proposed GA.

\section{Problem Codification}

A solution candidate of the optimal coordination problem is modeled as a vector of $n$ positions. Each entry of the vector corresponds to a $T M S_{i}$ for a relay. In this case, the length of the vector corresponds to the number of OCRs within the microgrid. Figure 2 illustrates an example of a candidate solution for a microgrid with $n$ relays. The TMS for relays 1 and 2 are set to 0.05 and 1 , respectively; while the one for relay $\mathrm{n}$ is set in 0.9 .

\begin{tabular}{|r|r|r|r|}
\hline TMS Relay 1 & TMS Relay 2 & $\ldots .$. & TMS Relay $n$ \\
\hline 0,05 & 1 & $\ldots .$. & 0,9 \\
\hline
\end{tabular}

Fig. 2. Example of a candidate solution of the GA.

\section{Initial Population and Fitness Evaluation}

The GA requires an initial population or set of candidate solutions that will be modified and improved over the next iterations according to a set of rules. In this case, the initial population is randomly generated taking into account the limits of the TMS given by (4).

Once a set of solution candidates is stablished, the fitness of each individual is evaluated. This is done using the results of a previous simulation of the system and verifying the coordination between the main and backup relays, as well as the total operating time. The previous simulation is done in the Digsilent Power Factory program. Therefore, a fitness value is associated to every individual of the initial population. This information is used in the tournament selection.

\section{Tournament Selection}

Once the objective function or fitness of each individual is evaluated, some individuals (parents) among the best are chosen to generate new candidate solutions (offspring). In this case, the section by tournament is implemented, which consists of randomly selecting two pairs of individuals and choosing the individual with the best performance. There are as many tournaments as there are number of individuals. The best individuals (in this case the parameters of the relays with better operating times) go to the next stage of crossover and mutation. Figure 3 illustrates the tournament selection.

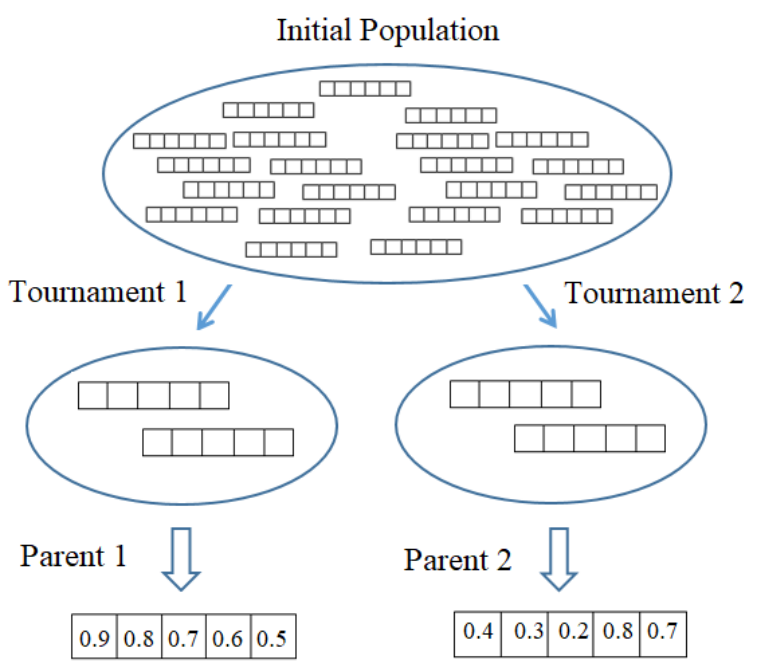

Fig. 3. Illustration of tournament selection.

\section{Crossover and Mutation}

Crossover or recombination is the stage of the algorithm in which parents exchange their genetic material to generate new individuals (offspring). On the other hand, mutation consists on altering one of the offspring, which allows, eventually, to escape from local optimal solutions incorporating diversification in the population. In this case, crossover and mutation occur with a given probability. The crossover is done by randomly selecting two parents of the winners of the tournament and crossing their bits, this generates individuals who share part of the genetic material of the parents. In the mutation stage, one of the offspring is randomly selected to subsequently change, with some probability, some of its bits. Once the crossover and mutation stages are finished, the population of offspring and parents is combined. Figure 3 illustrates the crossover stage for a microgrid with five OCRs.

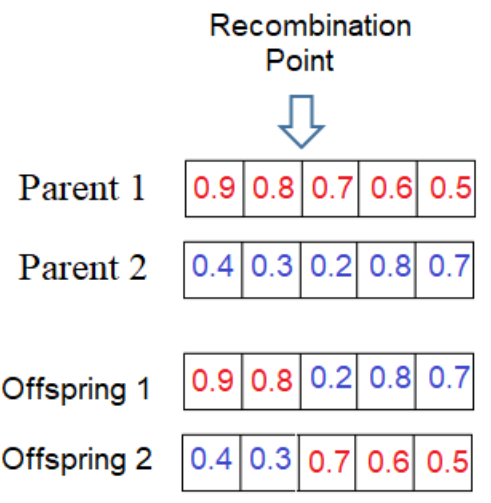

Fig. 3. Illustration of crossover. 


\section{New Generation}

For the selection of the new population, the fitness of each individual is used. The new generation begins to be formed by individuals with better objective function until they finish selecting half of the combined population of parents and children, the other half must be discarded. Finally, there are basically two stopping criteria: the total number of iterations or a maximum number of iterations without an improvement in individuals. If either of these two criteria is met, the algorithm stops. Figure 5 illustrates the creation of a new generation from the combined population of parents and offspring.

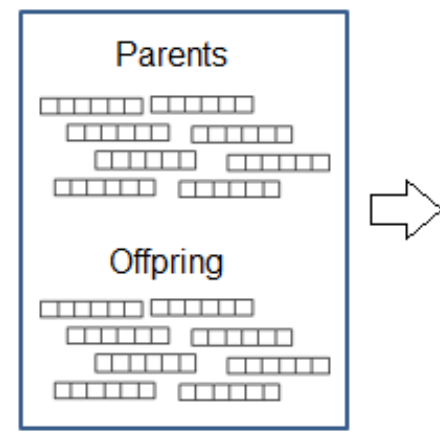

$2 n$ individuals

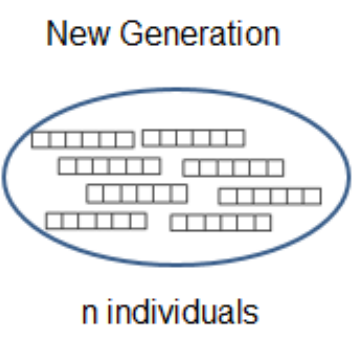

Fig 4. Illustration of a new generation.

\section{TESTS AND RESULTS}

To prove the effectiveness of the proposed approach, a benchmark IEC microgrid that integrates different DG technology types was considered. The parameters of the microgrid, depicted in Figure 5, can be consulted in [17]. The test system was implemented in DIgSILENT PowerFactory. The coordination time between main and backup relays, denoted as MCT, was set to 0.2 seconds.

For the operation time of the relays, IEC Normal Inverse curves are considered. The relays are named with numbers from 1 to 15 preceded by the letter $R$, the location of each relay is shown in Figure 5. In the cases of faults under analysis, after the $\mathrm{R}$ the letter $\mathrm{P}$ or $\mathrm{B}$ is assigned to indicate whether the relay is main or backup, respectively.

To adjust the parameters of the GA, different tests were performed varying the parameters of the algorithm. The combination of parameters that presented the best results after adjusting them are: population 100, generations 1000, crossing rate of 0.7 and mutation rate of 0.3 . The CB-LOOP1 and CB-LOOP2 were considered open.

Five faults were considered in the operational scenarios under analysis. Fault 1 occurs on line DL-5. Fault 2 occurs on line DL-4. Fault 3 is presented on the DL-2 line. Fault 4 is presented on DL-1 line. Finally, Fault 5 is presented on line DL-3. In this work, three modes of operation of the microgrid were analyzed. In operation mode 1 , the microgrid is connected to the main network while all DG units are disconnected. In operating mode 2, all DG units operate in the microgrid together with the main network. In operation mode 3 , the microgrid operates in island mode and the load is supplied by the DG units. The short circuit levels in every operation mode for every fault can be consulted in [9]. The results of the protection coordination are presented in Table I and Table II.

Table I presents the results of the OCR coordination obtained with the GA for the three modes of operation analyzed. The $T M S_{i}$ of each relay and the value of the objective function (1) in each mode of operation are presented. The results show that the shortest operating times are in mode 1 and that the times of mode 2 and 3 are very similar. The low operating times in mode 1 are because in this case the network has a radial topology and the current flow is given from the main network to the load. Modes 2 and 3 have longer operating times because in these cases the coordination is more complex due to the presence of the DG units, which generate bidirectional power flows. In addition, the GA delivers some equal parameters in all three modes of operation, this occurs in relays R2, R3, R10 and R12. The results presented in Table II show the operating times of the main and backup relays for each fault. In all three modes of operation, the proposed model guarantees coordination between the main and backup relays. In addition, in all the failures evaluated, it is evident that the main relays have a rapid response to clear the fault.

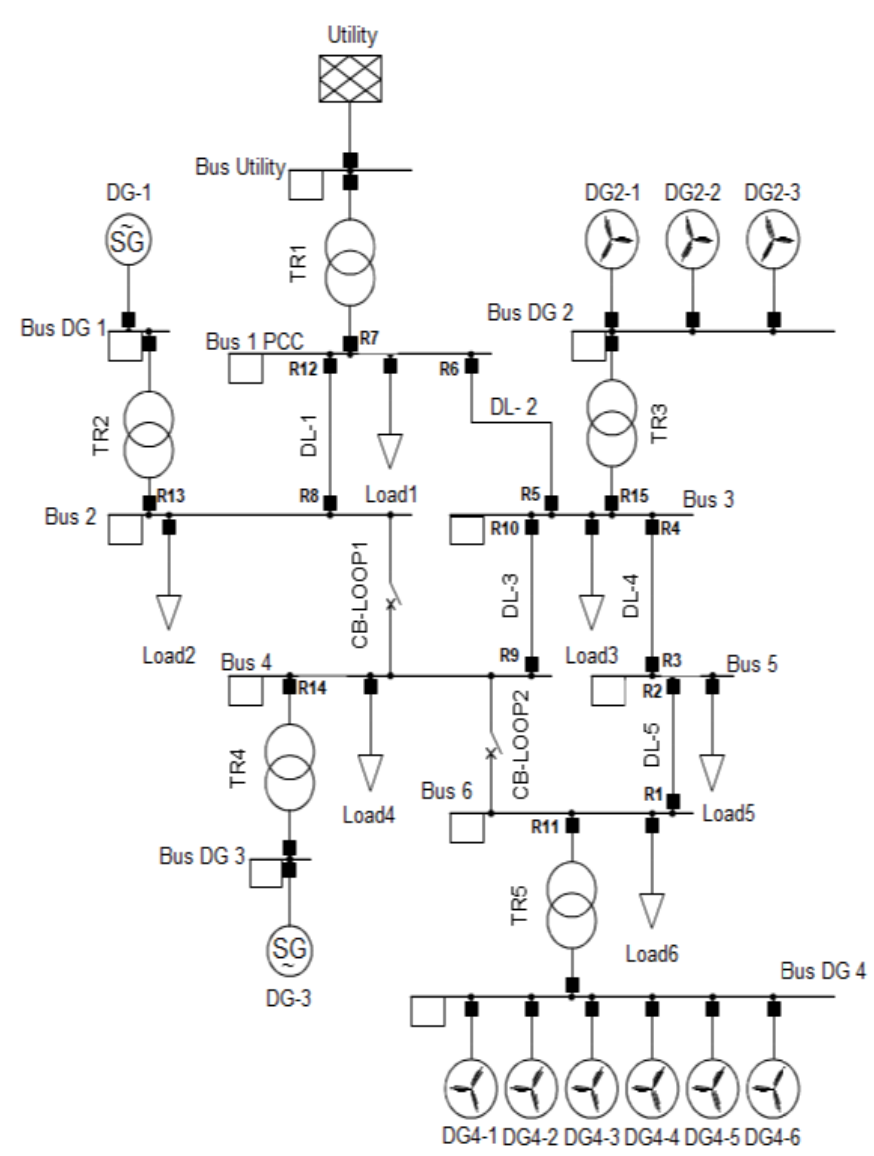

Fig. 5. Benchmark IEC microgrid. 
Table I

TMS for Different Operational Modes

\begin{tabular}{|c|c|c|c|}
\hline Relay & Mode 1 & Mode 2 & Mode 3 \\
\hline R1 & 0.5910 & 0.1580 & 0.1580 \\
\hline R2 & 0.1000 & 0.1000 & 0.1000 \\
\hline R3 & 0.1000 & 0.1000 & 0.1000 \\
\hline R4 & 0.1858 & 0.1928 & 0.1714 \\
\hline R5 & 0.3318 & 0.1147 & 0.1701 \\
\hline R6 & 0.2816 & 0.2744 & 0.1378 \\
\hline R7 & 0.2005 & 0.1930 & 0.6459 \\
\hline R8 & 0.9078 & 0.1513 & 0.1822 \\
\hline R9 & 0.4318 & 0.1000 & 0.1000 \\
\hline R10 & 0.1000 & 0.1000 & 0.1000 \\
\hline R11 & 0.6791 & 0.1649 & 0.1900 \\
\hline R12 & 0.1000 & 0.1000 & 0.1580 \\
\hline R13 & 0.3239 & 0.1598 & 0.1000 \\
\hline R14 & 0.7315 & 0.1221 & 0.1000 \\
\hline R15 & 0.2826 & 0.1166 & 0.1714 \\
\hline T(S) & 4.9 & 12.91 & 12.83 \\
\hline
\end{tabular}

Table II

Operation Time for Different Operational Modes

\begin{tabular}{|c|c|c|c|c|}
\hline Fault & Relay & $\begin{array}{l}\text { Mode } \\
1\end{array}$ & Mode 2 & Mode 3 \\
\hline \multirow{4}{*}{$\mathrm{F} 1$} & RP1 & & 0.5134 & 0.5134 \\
\hline & RP2 & 0.2331 & 0.2156 & 0.2800 \\
\hline & RB4 & 0.4331 & 0.4157 & 0.4800 \\
\hline & RB13 & & 0.7135 & 0.7135 \\
\hline \multirow{5}{*}{$F 2$} & RP3 & & 0.3446 & 0.3446 \\
\hline & RP4 & 0.3880 & 0.3624 & 0.4496 \\
\hline & RB1 & & 0.5444 & 0.5444 \\
\hline & RB6 & 0.5880 & 0.5624 & 0.6496 \\
\hline & RB15 & & 0.5623 & 0.6496 \\
\hline \multirow{5}{*}{$F 3$} & RP5 & & 0.3034 & 0.4500 \\
\hline & RP6 & 0.5083 & 0.4819 & 0.6211 \\
\hline & RB7 & 0.7084 & 0.6819 & 0.8213 \\
\hline & RB8 & & 0.6820 & 0.8213 \\
\hline & RB15 & & 0.6670 & 0.7705 \\
\hline \multirow{5}{*}{ F4 } & RP8 & & 0.6512 & 0.7842 \\
\hline & RP12 & 0.2088 & 0.1989 & 0.2851 \\
\hline & RB5 & & 0.3989 & 0.4850 \\
\hline & RB7 & 0.9521 & 0.9965 & \\
\hline & RB11 & & 0.8512 & 0.9839 \\
\hline \multirow{5}{*}{ F5 } & RP9 & & 0.4304 & 0.4304 \\
\hline & RP10 & 0.2331 & 0.2117 & 0.2660 \\
\hline & RB6 & 0.6564 & 0.6578 & 0.7942 \\
\hline & RB14 & & 0.6303 & 0.6303 \\
\hline & RB15 & & 0.8368 & 0.8855 \\
\hline
\end{tabular}

\section{CONCLUSION}

Protection coordination is one of the key issues in microgrid planning and operation. Since microgrids are expected to operate in various operational modes or topologies, classical approaches to protection coordination based on unidirectional power flows turned out not to be reliable. This paper presented an approach for the optimal coordination of overcurrent relays in microgrids. The main advantage of the proposed approach lies in its flexibility to obtain an optimal coordination scheme for several operation modes. A genetic algorithm was used to solve the proposed coordination model. Several tests were performed on a IEC benchmark microgrid considering different types of DG units under three operational modes. The algorithm was able to find a coordination scheme that minimizes the total operational time of the relays while keeping the coordination between main and back up relays. Simulations show that the coordination time is highly dependent on the operation mode. The shortest operating times were obtained in operation model 1 , in which the network presented a single source and unidirectional power flows. In operation modes 2 and 3, the coordination times were higher since the coordination is more complex due to the presence of the DG units and bidirectional power flows. In all three modes of operation, the proposed model guaranteed coordination between the main and backup relays.

\section{ACKNOWLEDGEMENTS}

The authors acknowledge the contribution of the Colombian Scientific Program within the framework of the so-called Ecosistema Científico (Contract No. FP44842- 218-2018).

\section{REFERENCES}

[1] S. A. Hosseini, H. A. Abyaneh, S. H. H. Sadeghi, F. Razavi, y A. Nasiri, "An overview of microgrid protection methods and the factors involved», Renew. Sustain. Energy Rev., vol. 64, pp. 174-186, oct. 2016, doi: 10.1016/j.rser.2016.05.089.

[2] A. Hooshyar y R. Iravani, «Microgrid Protection», Proc. IEEE, vol. 105, n. ${ }^{\circ} 7$, pp. 1332-1353, jul. 2017, doi: 10.1109/JPROC.2017.2669342.

[3] J. S. Giraldo, J. A. Castrillon, J. C. Lopez, M. J. Rider, y C. A. Castro, «Microgrids Energy Management Using Robust Convex Programming», IEEE Trans. Smart Grid, vol. 10, n. ${ }^{\circ}$ 4, pp. 4520-4530, jul. 2019, doi: 10.1109/TSG.2018.2863049.

[4] S. Parhizi, H. Lotfi, A. Khodaei, y S. Bahramirad, «State of the Art in Research on Microgrids: A Review», IEEE Access, vol. 3, pp. 890-925, 2015, doi: 10.1109/ACCESS.2015.2443119.

[5] Z. Li, M. Shahidehpour, F. Aminifar, A. Alabdulwahab, y Y. Al-Turki, «Networked Microgrids for Enhancing the Power System Resilience», Proc. IEEE, vol. 105, n. ${ }^{\circ}$ 7 , pp. 1289-1310, jul. 2017, doi: 10.1109/JPROC.2017.2685558. 
[6] B. Yan, P. B. Luh, G. Warner, y P. Zhang, «Operation and Design Optimization of Microgrids With Renewables», IEEE Trans. Autom. Sci. Eng., vol. 14, n. ${ }^{\circ}$ 2, pp. 573-585, abr. 2017, doi: 10.1109/TASE.2016.2645761.

[7] H. E. Farag, M. M. A. Abdelaziz, y E. F. El-Saadany, «Voltage and Reactive Power Impacts on Successful Operation of Islanded Microgrids», IEEE Trans. Power Syst., vol. 28, n. ${ }^{\circ}$ 2, pp. 1716-1727, may 2013, doi: 10.1109/TPWRS.2012.2223491.

[8] H. H. Zeineldin, Y. A.-R. I. Mohamed, V. Khadkikar, y V. R. Pandi, «A Protection Coordination Index for Evaluating Distributed Generation Impacts on Protection for Meshed Distribution Systems», IEEE Trans. Smart Grid, vol. 4, n. ${ }^{\circ} 3$, pp. 1523-1532, sep. 2013, doi: 10.1109/TSG.2013.2263745.

[9] S. D. Saldarriaga-Zuluaga, J. M. López-Lezama, y N. Muñoz-Galeano, «Optimal Coordination of Overcurrent Relays in Microgrids Considering a Non-Standard Characteristic», Energies, vol. 13, n. ${ }^{\circ}$ 4, p. 922, ene. 2020, doi: 10.3390/en13040922.

[10] H. M. Sharaf, H. H. Zeineldin, y E. El-Saadany, «Protection Coordination for Microgrids With GridConnected and Islanded Capabilities Using Communication Assisted Dual Setting Directional Overcurrent Relays», IEEE Trans. Smart Grid, vol. 9, n. ${ }^{\circ} 1$, pp. 143-151, ene. 2018, doi: 10.1109/TSG.2016.2546961.

[11] K. A. Saleh, H. H. Zeineldin, y E. F. El-Saadany, «Optimal Protection Coordination for Microgrids Considering $\mathrm{N} \$$-\$1 Contingency», IEEE Trans. Ind. Inform., vol. 13, n. ${ }^{\circ}$ 5, pp. 2270-2278, oct. 2017, doi: 10.1109/TII.2017.2682101.

[12] M. N. Alam, «Adaptive Protection Coordination Scheme Using Numerical Directional Overcurrent Relays», IEEE Trans. Ind. Inform., vol. 15, n. ${ }^{\circ} 1$, pp. 64-73, ene. 2019, doi: 10.1109/TII.2018.2834474.

[13] S. T. P. Srinivas y K. S. Swarup, «Optimal Protection Coordination for Microgrid Considering DG and Line Outages Using IWO-ILP Technique», en 2018 20th National Power Systems Conference (NPSC), 2018, pp. 1-6, doi: 10.1109/NPSC.2018.8771734.

[14] Y. Damchi, H. R. Mashhadi, J. Sadeh, y M. Bashir, «Optimal coordination of directional overcurrent relays in a microgrid system using a hybrid particle swarm optimization», en 2011 International Conference on Advanced Power System Automation and Protection, 2011, vol. 2, pp. 1135-1138, doi: 10.1109/APAP.2011.6180976.

[15] P. Ray, D. P. Mishra, y S. R. Arya, «An Intelligent Approach for Protection Coordination of Overcurrent Relay in Smartgrid», en 2019 IEEE 1st International Conference on Energy, Systems and Information Processing (ICESIP), 2019, pp. 1-6, doi: 10.1109/ICESIP46348.2019.8938354.
[16] H. R. Baghaee, M. Mirsalim, G. B. Gharehpetian, y H. A. Talebi, «MOPSO/FDMT-based Pareto-optimal solution for coordination of overcurrent relays in interconnected networks and multi-DER microgrids», Transm. Distrib. IET Gener., vol. 12, n. ${ }^{\circ}$ 12, pp. 28712886, 2018, doi: 10.1049/iet-gtd.2018.0079.

[17] S. Kar, S. R. Samantaray, y M. D. Zadeh, «Data-Mining Model Based Intelligent Differential Microgrid Protection Scheme», IEEE Syst. J., vol. 11, n. ${ }^{\circ}$ 2, pp. 1161-1169, jun. 2017, doi: 10.1109/JSYST.2014.2380432. 